\section{IT-Verträge auf Safe-Harbor screenen!}

Praxen und Kliniken, die mit einem ITHersteller einen Auftragsdatenverarbeitungsvertrag geschlossen haben, sollten dringend ihre Verträge prüfen, so der Bundesverband Gesundheits IT (bvitg). Der Grund ist ein Urteil des EuGH zum Safe-Harbor-Abkommen, das seit dem Jahr 2000 den Datentransfer zwischen US-Unternehmen und europäischen Anbietern regelt. Nachdem der EuGH dieses Abkommen nun für ungültig erklärt habe, sei damit auch die Einhaltung der datenschutzrechtlichen Mindeststandards bei Safe-Harbor-Verträgen nicht mehr gegeben, und dafür hafte der ärztliche Leistungserbringer als Dateninhaber. Rebecca Höhl

\section{Medical Office mit neuen Statistikfunktionen}

Indamed hat die Statistikfunktion im „Medical Office“ überarbeitet und dabei die Abfragefunktion verfeinert. Eine Baumstruktur ermögliche es, eigene Abfrageschablonen anzulegen und alle gewünschten Punkte des Krankenblatts und der Stammdaten in die Auswertung einzubeziehen, berichtet das Unternehmen. So könnten Statistiken über Patienten eines bestimmten Alters und Geschlechts mit bestimmten Erkrankungen ermittelt werden. Auf Tastendruck sind die Ergebnisse zudem als CSV-Dateien auslesbar und können damit für eine Weiterverarbeitung etwa im Controlling besser genutzt werden. Die Statistikfunktion ist Teil der Basisversion der Software und steht somit allen Anwendern direkt zur Verfügung.

Rebecca Höh

\section{Neue Formulare in der Praxissoftware}

Für Muster 1 (AU-Bescheinigung) und Muster 52 (Anfrage bei Fortbestehen der $\mathrm{AU})$ sind neue Vordrucke in den KBV-zertifizierten Softwaresystemen hinterlegt. Außerdem fällt Muster 17 (Bescheinigung für die Krankengeldzahlung) komplett weg, da die AU-Bescheinigung und das Krankengeldformular zum 1. Januar zusammengelegt wurden. Die Praxissoftware muss die Teams aber auch bei der Bedruckung von Formularen beziehungsweise bei der Abrechnung innerhalb der ambulanten spezialfachärztlichen Versorgung (ASV) unterstützen. eines Patienten-Smartphones mit dem Praxisnetzwerk eine Schadsoftware in die Praxis-EDV eingeschleppt wird, die dann deren Funktionen beeinträchtigt oder gar sensible Patientendaten ausspioniert.

\section{Besonders kritisch sind Datei-Importe}

Ein erhebliches Risiko birgt dabei der Import von Dateien, auch wenn diese etwa per E-Mail oder über SpeicherSticks übertragen werden. Denn leider sind die Zeiten längst vorbei, in denen noch die Faustregel galt, dass nur ausführbare Programmdateien Malware transportieren können. Durch sogenannte aktive Inhalte nisten sich Computerviren \& Co. längst auch in Wordund Excel-Dateien, PDF oder sogar Bilddateien ein. Dass ein Hacker die Zeit und Mühe investiert, dies auch bei den spezialisierten Datenformaten von $\mathrm{Ge}$ sundheits-Apps zu erreichen, ist zwar unwahrscheinlicher, aber technisch keineswegs unmöglich. Symantec-Experte O’Brien führt aus: „Bei der Einschätzung von Malware-Bedrohungen lohnt sich immer ein Blick auf die wirtschaftliche Dimension." Zwar gibt es in Arztpraxen kaum Zugänge zu Zahlungssystemen oder Bankkonten abzugreifen, jedoch bietet die Sensibilität der hier gespeicherten Daten zum Beispiel Erpressungspotenzial.

\section{Medienbruch aus \\ Datensicherheitsgründen}

Doch wie sollen Ärzte auf entsprechende Anfragen technikaffiner Patienten reagieren? Schließlich spielt gerade im Kontext von Gesundheitsvorsorge und Sportbegleitung der Servicegedanke eine immer wichtigere Rolle im Verhältnis zwischen Ärzten und Patienten.

Auch wenn es aus technischer Perspektive wenig überzeugend klingt, bietet den besten Schutz vor Malware ein absichtlicher Medienbruch. Im Zweifel lohnt es sich aus Sicherheitserwägungen, die Patienten zu bitten, Auswertungen von Gesundheits-Apps oder Messwerte ihrer Smart-Devices auf Papier auszudrucken und in die Sprechstunde mitzubringen oder vorab in der Praxis einzureichen. In der Regel wird eine komplette Übernahme aller Daten in das Soft- waresystem der Praxis ohnehin nicht erforderlich sein. Zumindest auf Sicht vermeiden Ärzte so die kritische Direktanbindung fremder Daten und Geräte an ihre Praxissysteme.

\section{Schnittstellen in Arbeit}

Es gibt allerdings erste Ansätze für gemeinsame Standards oder einheitliche Datenschnittstellen für gesundheitsbezogene Smartphone-Anwendungen. „Vorreiter ist hier mal wieder Apple, das mit ,Healthkit' auf iPhones und iPads eine zentrale Sammelstelle für Gesundheitsdaten bereitstellt", berichtet Dr. Andreas Zollmann, Geschäftsführer des auf Macintosh-Praxissoftware spezialisierten Jenaer Anbieters Zollsoft. Die gesicherte Übernahme der HealthkitDaten in das Praxissystem sei für die Zukunft denkbar, sofern genügend Ärzte Interesse bekunden. Bis es soweit ist, sollten Praxen und Krankenhäuser in Sachen Datensicherheit lieber konservativ agieren und allzu fordernden Patienten bei Bedarf die Hintergründe dieser grundsätzlichen Erwägungen vermitteln. Denn letztlich dient auch dies dem - informationstechnischen - Wohl des Patienten.

Hannes Rügheimer

\section{Gesundheits-Apps im Test \\ — Weltweit rund 380.000 Apps zum Thema Gesundheit, Medizin, Life- style und Ernährung hat das Univer- sitätsklinikum Freiburg in einer Stu- die zu Gesundheits-Apps gezählt. \\ _ Mit einigen wenigen Ausnahmen sind die Gesundheits-Apps in den App-Stores laut der Studie keine Medizinprodukte. \\ _ Eine CE-Kennzeichnung sage nichts über die Qualität oder den Nutzen der App aus, warnen die Forscher. Die CE-Kennung besagt nämlich nichts anderes, als dass ein Produkt den Anforderungen entspricht, die Herstellern in der EU auferlegt sind, um es hier verkaufen zu können. Zu- dem bestätigt der Hersteller in eige- ner Verantwortung, dass er die CE- Bestimmungen erfüllt.}

Die Studie im Web:

http://tinyurl.com/hdzwvjj 